\title{
Correction to: Coronal and sagittal laxity affects clinical outcomes in posterior-stabilized total knee arthroplasty: assessment of well-functioning knees
}

\author{
Toshifumi Watanabe $^{1,2}$ (D) Hideyuki Koga ${ }^{2} \cdot$ Hiroki Katagiri $^{2} \cdot$ Koji Otabe $^{2} \cdot$ Yusuke Nakagawa $^{2} \cdot$ Takeshi Muneta $^{2} \cdot$ \\ Ichiro Sekiya ${ }^{2} \cdot$ Tetsuya Jinno ${ }^{2}$
}

Published online: 28 May 2019

(c) European Society of Sports Traumatology, Knee Surgery, Arthroscopy (ESSKA) 2019

\section{Correction to: \\ Knee Surgery, Sports Traumatology, Arthroscopy https://doi.org/10.1007/s00167-019-05500-8}

Unfortunately, the co-author "Hiroki Katagiri' was omitted in the original publication from the author group. The author name is added here. The original article has been corrected.

Publisher's Note Springer Nature remains neutral with regard to jurisdictional claims in published maps and institutional affiliations.
The original article can be found online at https://doi.org/10.1007/ s00167-019-05500-8.

Toshifumi Watanabe

toshi-w@dokkyomed.ac.jp

Hideyuki Koga

koga.orj@tmd.ac.jp

Hiroki Katagiri

katagiri.orj@tmd.ac.jp

Koji Otabe

otabe.arm@tmd.ac.jp

Yusuke Nakagawa

ynakagawa.orj@tmd.ac.jp

Takeshi Muneta

munetaorj3255@gmail.com
Ichiro Sekiya

sekiya.orj@tmd.ac.jp

Tetsuya Jinno

jinnot@dokkyomed.ac.jp

1 Second Department of Orthopaedic Surgery, Dokkyo Medical University Saitama Medical Center, 2-1-50 Minami-Koshigaya, Koshigaya-shi, Saitama 343-8555, Japan

2 Department of Orthopaedic Surgery, Tokyo Medical and Dental University Hospital, 1-5-45 Yushima, Bunkyo-ku, Tokyo 113-8510, Japan 